\title{
MÉTODOS DE ESTUDIO Y RENDIMIENTO ACADÉMICO EN LOS ESTUDIANTES DE PRIMER SEMESTRE DE CONTADURÍA PÚBLICA DE LA UFPS SECCIONAL OCAÑA, MEDIANTE LA PROPUESTA DE FERNANDEZ POZAR
}

\section{METHODS OF STUDY AND ACADEMIC PERFORMANCE IN STUDENTS OF THE FIRST SEMESTER OF PUBLIC ACCOUNTING OF THE UFPS SECTIONAL OCAÑA, BY THE PROPOSAL OF FERNANDEZ POZAR}

\author{
MSc. Sandra Olimpia Quintana Arevalo ${ }^{\mathrm{a}}$, MSc. Javier Numa Numa ${ }^{\mathrm{b}}$, MSc. Ricardo Andrés \\ García León $^{c}$
}

\begin{abstract}
${ }^{a}$ Universidad Francisco de Paula Santander Ocaña, grupo de investigación ROTA, Vía Acolsure Sede el Algodonal, Ocaña - Norte de Santander, Colombia, soquintanaa@ufpso.edu.co

${ }^{\mathrm{b}}$ Universidad Francisco de Paula Santander Ocaña, Vía Acolsure Sede el Algodonal, Ocaña Norte de Santander, Colombia,jnuman@ufpso.edu.co

${ }^{c}$ Universidad Francisco de Paula Santander Ocaña, Grupo de Investigación INGAP, Vía Acolsure Sede el Algodonal, Ocaña - Norte de Santander, Colombia, ragarcial@ufpso.edu.co
\end{abstract}

\begin{abstract}
Resumen: La presente investigación buscó mediante la aplicación del Inventario de Hábitos de Estudio de F.F. Pozar (IHE) describir los hábitos de estudio de los estudiantes de contaduría pública al inicio de su carrera universitaria (I semestre) y después de un año de estudio en la universidad Francisco de Paula Santander Ocaña, asimismo se identificó en cada estudiante el rendimiento académico de cada semestre, a través del promedio ponderado del semestre, teniendo en cuenta los valores que se correlacionaron con los resultados de los estudiantes en el IHE; por otra parte, se caracterizaron los métodos de estudio usados por los estudiantes a través de un cuestionario abierto que permitió describir y conocer la capacidad instalada en los estudiantes en relación a su conducta de estudio. El diseño metodológico utilizado es de tipo cuantitativo correlacional, el cual tiene como propósito mostrar o examinar la relación entre variables o resultados que se puedan presentar entre las variables estudiadas en esta investigación en unos momentos determinados, a través de la aplicación del Inventario de Hábitos de Estudio (IHE) y su relación con el rendimiento académico. Los resultados demostraron que no existe relación entre el inventario y el rendimiento académico de los estudiantes, debido a que las tecnologías, la sociedad y otros factores han influenciado en los cambios de los hábitos de estudio.
\end{abstract}


Palabras clave: Hábitos de estudio, IHE, Educación superior, Metodología

Abstract: The present research searched through the application of the Inventory of Habits of Study of F.F. Pozar (IHE) to describe the study habits of students of public accounting at the beginning of their university career (I semester) and after a year of study at the university Francisco de Paula Santander Ocaña, also identified in each student academic performance Of each semester, through the weighted average of the semester, taking into account the values that were correlated with the students' results in the IHE; On the other hand, the study methods used by the students were characterized by an open questionnaire that allowed to describe and to know the installed capacity in the students in relation to their study behavior. The methodological design used is a quantitative correlational type, whose purpose is to show or examine the relationship between variables or results that may be present among the variables studied in this research at a given time point, through the application of the Inventory of Habits of Study (IHE) and its relation with academic performance. The results showed that there is no relationship between the inventory and academic performance of students, because technologies, society and other factors have influenced changes in study habits.

Keywords: Habits of study, IHE, Higher Education, Methodology.

\section{INTRODUCCIÓN}

La Revista Ingenio es una publicación anual de carácter interdisciplinario para la difusión de trabajos originales en cualquier área de la Ingeniería, ciencias agrarias y del ambiente, ciencias administrativas y económicas, educación, artes y humanidades.

La investigación realizada hace parte del macroproyecto de la línea de investigación Evaluación y Calidad de la Educación, de la Maestría en práctica Pedagógica de la Universidad Francisco de Paula Santander, que aborda las áreas temáticas de Hábitos de Estudio y Rendimiento Académico de los estudiantes de la UFPS Seccional Ocaña.

Los hábitos de estudio se constituyen en un elemento fundamental en la conducta de estudio. Sobre el tema, Quelopana (1999) explica que el hábito es una costumbre para hacer algo como natural, el hábito de estudiar es la práctica de estudio sin necesidad de ordenar de nuevo para hacerlo; una persona no nace con el hábito; éste se adquiere y se aprende; todo estudiante requiere y necesita desarrollarlo. Kancepolski y Ferrante (1992) definen que los hábitos de estudio tienen por fin, lograr el aprendizaje, entendido este desde una postura cognitivista como un proceso de comprensión, integración, interacción, asimilación y acomodación de la información. Ortega (2012) cita, en su trabajo de investigación, los autores que se presentan a continuación, y que hacen referencia sobre los hábitos de estudio: "Poves (2001) señala que el hábito de estudio es una acción que se realiza todos los días aproximadamente a la misma hora, la reiteración de ésta conducta en el tiempo va generando un mecanismo inconsciente. Hernández (1988) son un conjunto de hábitos de trabajo intelectual que afectan a las funciones de motivación, condiciones físicas y destrezas instrumentales básicas para el estudio; cada una de éstas proporciona elementos que permiten un adecuado desenvolvimiento del estudiante en el que hacer educativo, así como en su 
contexto personal. Martínez, Pérez \& Torres (1999) definen a los hábitos de estudio como la práctica constante de las mismas actividades; se requiere de acciones cotidianas, las cuales serán con el tiempo un hábito afectivo siempre y cuando sean asumidas con responsabilidad, disciplina y orden. Belaunde (1994) considera que el concepto de hábito de estudio está referido al modo como el individuo se enfrenta cotidianamente a su quehacer educativo, es la costumbre natural de procurar aprender permanentemente, lo cual implica la forma en el que el individuo se organiza en cuanto a tiempo, espacio, técnica y métodos, concretos que utiliza para estudiar".

La perspectiva teórica de Pozar (2002), coherente con los planteamientos anteriores, define los hábitos de estudio como las actividades que utiliza un estudiante en forma repetitiva para generar aprendizaje y garantizar el éxito en las tareas escolares, siempre y cuando cuente con condiciones ambientales para el estudio, desarrolle una apropiada planeación del estudio, adecuada utilización de materiales y asimilación de contenidos, con lo cual logrará alcanzar la meta propuesta. La investigación desarrollada, trabajó desde esta perspectiva y valoró los hábitos de estudio desde la propuesta de Pozar: Inventario de Hábitos de Estudio (IHE); el I.H.E. mide los hábitos de estudio de los alumnos por medio de cuatro escalas fundamentales, a las que se añade una escala de sinceridad, estas son: a) Escala I: Condiciones ambientales del estudio. b) Escala II: Planificación del estudio. c) Escala III: Utilización de materiales. d) Escala IV: Asimilación de contenidos. Otra variable, objeto de estudio en la presente investigación, fue el rendimiento académico, el cual es definido por Villarroel (1989), como las calificaciones que obtuvo el estudiante por el aprendizaje adquirido en una asignatura, materia o curso. Pizarro (1985), señala que el rendimiento académico es un indicador del éxito frente a las demandas de la formación docente, es una medida de las capacidades respondientes en forma estimativa, es decir lo que una persona ha aprendido como consecuencia de un proceso de formación.

Trascendiendo el tema de los hábitos de estudio es importante para la investigación, conocer el método de estudio que los estudiantes reportan al llegar al sistema de educación superior. Ante esta situación y panorama presentado en relación con la conducta de estudio, método y hábitos de estudio y el rendimiento académico del estudiante, se motiva las siguientes preguntas de investigación:

- ¿Existe correlación entre el rendimiento académico y los hábitos de estudio de los estudiantes de Contaduría Pública una vez inician su estudio universitario?

- ¿Existe correlación entre el rendimiento académico y los hábitos de estudio de los estudiantes de Contaduría Pública después de un año de haber iniciado sus estudios universitarios?

- ¿Cómo se caracterizan los hábitos de estudio de los estudiantes de Contaduría Pública, a partir de la propuesta de Pozar IHE?

Justificar el estudio que se ha realizado y que ha permitido analizar la relación entre hábitos de estudio y rendimiento académico, es un elemento importante en el contexto universitario, ante la necesidad de trabajo independiente que el estudiante debe realizar para cumplir con los objetivos de los diferentes programas de asignaturas de una carrera universitaria; además, porque los 
resultados de la investigación brindan información objetiva sobre los hábitos de estudio de los estudiantes y sobre su propio método de estudio, lo cual permitirá no solo a la institución sino también a los docentes tomar decisiones que posibiliten la calidad y mejoramiento académico de los estudiantes

En la presente investigación, se establecen los hábitos de estudio de los estudiantes del programa de Contaduría Pública de la Universidad Francisco de Paula Santander seccional Ocaña, para lo cual se aplicaron dos Inventarios de Hábitos de Estudio IHE, propuestos por Fernández Pozar, a cada uno de los elementos que hicieron parte de la población en estudio.

El primer inventario fue aplicado en el segundo semestre del año 2014, a una población de 54 estudiantes de Contaduría Pública, cuando los estudiantes iniciaban su ciclo profesional. Un segundo inventario se aplicó en el segundo semestre 2015, a esos mismos estudiantes, lo cuales ya cursaban el segundo semestre académico de la carrera correspondiente. Esta población se redujo a un total de 34 , debido a que se presentó una deserción de 20 estudiantes.

\section{METODOLOGÍA}

El Diseño de la investigación es correlacional, tal como lo propone Hernández (1991, p.62), su propósito, “es medir el grado de relación que existe entre dos o más variables en un contexto en particular'. Se orienta a la perspectiva empírico-analítica, enfatiza el contexto de justificación o contrastación de hipótesis, siendo no experimental o ex post-facto, en lo que hacemos es "observar fenómenos tal y como se da en su contexto natural para después analizarlos" (Hernández, et al. 2004, p.184). La investigación correlacional, como lo expresa Salkind (citado por Bernal. 2006. P. 111) Esta investigación tiene como propósito mostrar o examinar la relación entre variables o resultados que se puedan presentar entre las variables estudiadas en esta investigación; en unos momentos determinados, a través de la aplicación del Inventario de Hábitos de Estudio (IHE) y su relación con el rendimiento académico. Otra utilidad es saber cómo se puede comportar una variable, conociendo el comportamiento de otra $\mathrm{u}$ otras variables relacionadas. (Vodniza, 2009. P. 78)

Para abordar el objetivo que refiere al tema de describir los métodos de estudio usado por los estudiantes, se usó un diseño de investigación descriptivo de tipo longitudinal ya que como lo cita Hernández, et al. 2004, buscan especificar las propiedades, las características y los perfiles importantes de personas, grupos, comunidades o cualquier otro fenómeno que se someta a un análisis; miden, evalúan o recolectan datos sobre diversos aspectos, dimensiones o componentes del fenómeno a investigar.

Los estudios longitudinales nos permiten analizar los cambios que pueda presentar alguna variable a través del tiempo y la relación que existe entre ellas para sí poder realizar inferencias que nos lleven a aceptar o rechazar nuestras hipótesis planteadas en nuestra investigación.

Ary, D., Cheser, L. y Razavieh, A. (1982), consideran que la investigación de carácter descriptivo permite precisar la naturaleza de una situación tal como existe en el momento dado en que se realiza el estudio.

La población que se tuvo en cuenta para la investigación fue de 54 estudiantes que iniciaron estudios superiores en el segundo semestre de 2014 en la universidad 
Francisco de Paula Santander, seccional Ocaña, Colombia., distribuidos así: 38 de la jornada diurna y 16 de la jornada nocturna, a quienes se les aplicó una primera medición cuando cursaban el primer semestre de su carrera. Un año después se aplicó una segunda medición, a la misma población inicial, para lo cual, se habían presentado 20 desertores, quedando un total de 34 estudiantes: 26 de la jornada diurna y 8 de la jornada nocturna.

El proceso de recolección de información se realizó a través del Instrumento Hábitos de Estudio (IHE) de Fernández Pozar, 10ª edición revisada y ampliada, Tea Ediciones Madrid, 2014 Esta es una prueba de estudio elaborada con un propósito básico: detectar hasta qué punto el estudiante conoce su oficio. El objetivo principal del IHE es definir las actitudes del estudiante hacia su trabajo. La utilización del IHE, presenta tres finalidades:

- Diagnosticar la naturaleza y grado de los hábitos, actitudes o condiciones con que el estudiante se enfrenta a su tarea de estudio específica.

- Pronosticar las consecuencias que, en orden al aprendizaje académico o a la formación cultural, cabe esperar del influjo de estos hábitos, independientemente de la incidencia de otras variables.

- Actuar, a partir del diagnóstico, en la dirección adecuada para modificar los hábitos defectuosos o favorecer la adquisición e incremento de los considerados beneficiosos.

El IHE consta de 90 elementos distribuidos en 5 escalas:

- Escala I. Condiciones ambientales del estudio: 18 elementos que reúnen los condicionamientos del ambiente que rodean al estudiante, como son los personales, ambiente físico, el comportamiento académico y el rendimiento.

- Escala II. Planificación del estudio: 12 elementos, que involucra horarios de todas las actividades, así como la organización de los materiales y elementos necesarios para el estudio.

- Escala III. Utilización de materiales: 15 elementos los cuales se relacionan con el manejo de los libros, las lecturas y los resúmenes.

- Escala IV. Asimilación de contenidos: 15 elementos que analizan el grado de memorización, como la personalización que influyen directamente en el trabajo personal y en equipo.

- Escala S. Sinceridad: 30 elementos repartidos en toda la prueba.

Para continuar con la interpretación de la prueba, F. Fernández Pozar (2014), propone en su Manual de IHE, unos criterios orientativos de clasificación de los eneatipos, los cuales se describen en la tabla siguiente:

Tabla 1. Clasificación de los eneatipos

\begin{tabular}{|c|c|c|c|c|c|}
\hline \multicolumn{5}{|c|}{ Eneatipos } & Calificación \\
\hline & & 9 & & & Excolente \\
\hline & 7 & - & 8 & & Bien \\
\hline 4 & - & 5 & - & 6 & Normal \\
\hline & 2 & - & 3 & & No satisfactorio \\
\hline & & 1 & & & Mal \\
\hline
\end{tabular}

Fuente: Inventario de Hábitos de Estudio de Pozar

El IHE, establece unos baremos de acuerdo con el tipo de escolaridad que presente la muestra seleccionada para la aplicación del Instrumento. En nuestro caso y de acuerdo con lo planteado por Pozar (2014), el baremo que se empleó para el análisis de la información fue el siguiente: Escolares de 17 - 19 años, varones y mujeres. 
Tabla 2. Baremo para la interpretación de los datos

\begin{tabular}{|c|c|c|c|c|c|c|}
\hline \multicolumn{7}{|c|}{ BAREMOS de escolares: 17-19 años, varones y mujeres ( 2do. De Bachillerato) } \\
\hline \multirow{2}{*}{$\begin{array}{c}\text { Eneatipo } \\
\text { (en) }\end{array}$} & \multicolumn{5}{|c|}{ Puntuación directa (PD) en escalas } & \multirow{2}{*}{$\begin{array}{c}\text { Eneatipo } \\
\text { (en) }\end{array}$} \\
\hline & I.COND.AMB. & II.PLAN ESTUD. & \begin{tabular}{|l} 
III. UTIL.MAT. \\
\end{tabular} & IV. ASIM.CONT. & S.SINC. & \\
\hline 9 & $31-33$ & $22-24$ & 24 & $29-30$ & $29-28$ & 9 \\
\hline 8 & $28-30$ & $20-21$ & $22-23$ & $27-28$ & $24-25$ & 8 \\
\hline 7 & 27 & 18-19 & 21 & 26 & 23 & 7 \\
\hline 6 & $25-26$ & $16-17$ & 20 & 25 & $21-22$ & 6 \\
\hline 5 & $22-24$ & 13-15 & 18-19 & $23-24$ & $19-20$ & 5 \\
\hline 4 & $20-21$ & $9-12$ & 15-17 & $21-22$ & $17-18$ & 4 \\
\hline 3 & 18-19 & $6-8$ & 13-14 & $\begin{array}{l}19-20 \\
\end{array}$ & $15-16$ & 3 \\
\hline 2 & 15-17 & 4-5 & 11-12 & $16-18$ & 13-14 & 2 \\
\hline 1 & $0-14$ & $0-3$ & $0-10$ & $0-15$ & $0-12$ & 1 \\
\hline $\mathrm{N}$ & & & 350 & & & $\mathrm{~N}$ \\
\hline Media & 22,77 & 12,97 & 17,78 & 22,86 & 19,27 & Media \\
\hline $\mathrm{Dt}$ & 4,71 & 5,35 & 3,95 & 3,73 & 3,71 & $\mathrm{dt}$ \\
\hline
\end{tabular}

Fuente: Inventario de Hábitos de Estudio de Pozar

Por último, empleando los datos arrojados por la aplicación del baremo escogido, se describe el perfil del estudiante, así:

Tabla 3. Perfil del estudiante

\begin{tabular}{|c|c|c|c|c|c|c|c|c|c|c|c|}
\hline \multicolumn{12}{|c|}{ PERFIL } \\
\hline \multirow{2}{*}{\multicolumn{2}{|c|}{ ESCALA }} & \multirow{3}{*}{$\begin{array}{l}\text { PD } \\
20\end{array}$} & \multirow{3}{*}{$\begin{array}{c}\text { Mal } \\
1 \\
0\end{array}$} & \multicolumn{2}{|c|}{ No satisfactorio } & \multicolumn{3}{|c|}{ Normal } & \multicolumn{2}{|c|}{ Bien } & \multirow{2}{*}{\begin{tabular}{|c|} 
Excelente \\
9
\end{tabular}} \\
\hline & & & & 2 & 3 & 4 & 5 & 6 & 7 & 8 & \\
\hline 1 & Condiciones ambientales & & & 0 & & مـ & 0 & 0 & 0 & $\circ$ & o \\
\hline$\|$ & Planificación & 4 & 0 & $\ll$ & 0 & 0 & 0 & 0 & 0 & 0 & 0 \\
\hline III & Utilización de materiales & 21 & 0 & 0 & 0 & o & 0 & & $\rightarrow$ & 0 & 0 \\
\hline IV & Asimilación de contenidos & 19 & 0 & 0 & $\circ$ & $\bullet$ & 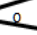 & 0 & 0 & 0 & 0 \\
\hline $\mathrm{s}$ & Sinceridad & 26 & 0 & 0 & 0 & 0 & 0 & 0 & 0 & 0 & $\longrightarrow$ \\
\hline
\end{tabular}

Fuente: Inventario de Hábitos de Estudio de Pozar

Adicionalmente al IHE de Fernández Pozar, Se aplicó un instrumento de preguntas abiertas a 80 estudiantes de la población de la primera aplicación, con el propósito de establecer claramente sus hábitos de estudio y su incidencia en el rendimiento escolar. Dicho instrumento constó de cinco (5) preguntas que indagaron: Cómo estudia, Cuáles son las estrategias o acciones más efectivas en su estudio, En qué lugar estudia, Con quién estudia y Para qué estudia Las hipótesis planteadas, fueron:

- Ho: Los hábitos de estudio, poco o nada influyen significativamente en el Rendimiento académico de los estudiantes

- H1: Los hábitos de estudio influyen significativamente en el Rendimiento académico de los estudiantes

Las limitaciones presentadas en la aplicación delas pruebas, fue la ausencia de los estudiantes en el momento de requerirlos, por lo que se realizaron los intentos necesarios hasta lograr su ubicación.

La descripción de los métodos ha de tener el suficiente detalle de manera que permita a cualquier investigador experimentado reproducirlo, esta descripción ha de seguir un orden lógico de forma que el lector pueda comprender cómo la manipulación descrita se ajusta al diseño experimental. La información debe describir lo siguiente:

- El diseño del experimento o investigación.

- La muestra.

- Las restricciones o limitaciones.

- Las técnicas.

- Los procedimientos.

- Los materiales.

- Las variables.

- El tratamiento estadístico.

\section{RESULTADOS}

A continuación, se presentan los resultados basados en el instrumento de F. F. Pozar:

\subsection{Estudiantes de Contaduría Pública jornada diurna primera aplicación.} Fueron 38 estudiantes, donde la sinceridad en las respuestas están dentro de los normal y lo excelente, superando el $80 \%$. El mejor hábito del grupo al ingresar a la universidad, es la utilización de materiales de estudio, que entre lo normal y lo excelente, asciende al 94\%, seguido de las condiciones ambientales en las que realizan su actividad 
académica, con un $89 \%$, la planificación de los estudios con un $84 \%$ y la asimilación de contenidos con casi el $82 \%$. Un mínimo porcentaje $(2.63 \%)$ alcanza la excelencia en todos los aspectos, menos en la asimilación de contendidos que es de cero. Se aprecia que el grupo ingresó con buenos hábitos de estudio.

3.1.2 Estudiantes de Contaduría Pública
jornada nocturna primera aplicación. El total de estudiantes fue de 16 , quienes ingresaron a la universidad con hábitos similares a los de la jornada diurna, superándolos en sus condiciones ambientales, seguido de la planificación del estudio y de la asimilación de contenidos. Con menos participación está la utilización de materiales de estudio. La sinceridad en las respuestas, es de una normalidad del, superando a los de la jornada diurna. La excelencia la alcanza el $6.25 \%$ en los dos primeros aspectos y en la sinceridad.

\subsubsection{Resultados depurados Primera} aplicación Estudiantes de Contaduría Pública jornada diurna. La depuración de los resultados consiste en no tener en cuenta los inventarios que presentaron sinceridad No Satisfactoria y Mala; por lo tanto la población quedó en 31, y los resultados indican que se mantiene normalidad en todos los hábitos de estudio.

\subsubsection{Resultados depurados Primera} aplicación Estudiantes de Contaduría Pública jornada nocturna. Depurada la información, la población pasó de 16 a 12 . Muestran debilidad en la planificación del estudio, lo cual indica que un $25 \%$ no satisfactorio, así mismo, un $16.67 \%$ en la asimilación de contenidos. La excelencia la alcanzan en tres aspectos: condiciones ambientales, planificación de estudios y sinceridad, todas en un $8.33 \%$. Lo demás está dentro de lo normal.
3.1.6 Estudiantes de Contaduría Pública jornada nocturna primera aplicación. La población fue de 26 estudiantes, quienes muestran el mismo comportamiento del grupo consolidado. En los primeros tres aspectos se logra un mínimo porcentaje de excelencia. Lo no satisfactorio de los hábitos de estudio, oscila entre el $7.69 \%$ y el $15.38 \%$; estos últimos en la asimilación de contendidos y en la sinceridad en las respuestas. El resto está dentro de lo normal.

\subsubsection{Estudiantes de Contaduría Pública jornada nocturna segunda aplicación. $\mathrm{La}$} población fue de 8 estudiantes, en los que pudo apreciar que ninguno respondió con mala sinceridad y sólo el $12.50 \%$ con sinceridad no satisfactoria, sin embargo, la excelencia en esta fue alta, $37.50 \%$. El $50 \%$ presenta normalidad en condiciones ambientales, planificación del estudio y asimilación de contenidos. El 37.5\% aplica muy bien las condiciones ambientales de estudio y el $12.50 \%$ en forma excelente la utilización de materiales.

\subsubsection{Resultados depurados Segunda aplicación Estudiantes de Contaduría} Pública jornada diurna. La población fue de 21 estudiantes y muestran una excelencia en la aplicación de los diferentes hábitos de estudio. Alto el porcentaje de los buenos hábitos en condiciones ambientales $(47.62 \% \%)$, utilización de materiales (38.10\%) y en asimilación de contenidos $(28.57 \%)$. Todos los hábitos tienen gran representación dentro de lo normal, especialmente la planificación del estudio que está en un $66.67 \%$. La sinceridad muestra una normalidad del $85.71 \%$. Los aspectos que presentan un índice importante en lo no satisfactorio, son la planificación del estudio y la asimilación de contenidos, con un $14.29 \%$ cada uno.

\subsubsection{Resultados depurados Segunda} aplicación Estudiantes de Contaduría 
Pública jornada nocturna. Esta población fue solo de 6 inventarios con respuestas sinceras. Los resultados indican excelencia del $16.67 \%$ en la utilización de materiales y $33.33 \%$ en sinceridad; alto este porcentaje. Los demás aspecto califican cero en excelencia. Alto el porcentaje de los buenos hábitos en condiciones ambientales $(33.33 \%)$, los demás se mantienen en el $16.67 \%$ a excepción de la sinceridad que marca cero. Los altos porcentajes están en todos los aspectos en la calificación normal, la cual va desde $50.00 \%$ hasta el $66.67 \%$ como es el caso de a planificación del estudio y la sinceridad. Ninguno de los hábitos es mal utilizado. En no satisfactorio están todos los aspectos en el $16.67 \%$ a excepción de la asimilación de contenidos que está en un $33.33 \%$.

\subsubsection{Resultados consolidados depurados} segunda aplicación diurno y nocturno. La excelencia es baja en todos los aspectos. Alto el porcentaje de los buenos hábitos en condiciones ambientales (44.44\%), utilización de materiales $(33.33 \%)$ y asimilación de contenidos (25.93\%). Los altos porcentajes están en todos los aspectos en la calificación normal; condiciones ambientales con el $44.44 \%$, planificación del estudio con $66.67 \%$, utilización de materiales con el $48.15 \%$, asimilación de contenidos con $51.85 \%$ y la sinceridad con el $81.48 \%$. En no satisfactorio están todos los aspectos, y oscilan entre $7.41 \%$ y el $18.52 \%$, este último porcentaje corresponde a la asimilación de contenidos.

3.1.11 Comparativo resultados depurados primera y segunda aplicación jornada diurna. El Perfil comparativo resultados depurados estudiantes jornada diurna, primera (línea azul) y segunda (línea roja) aplicación, se muestra en la gráfica siguiente:
Tabla 4. Perfil comparativo resultados depurados primera y segunda aplicación jornada diurna

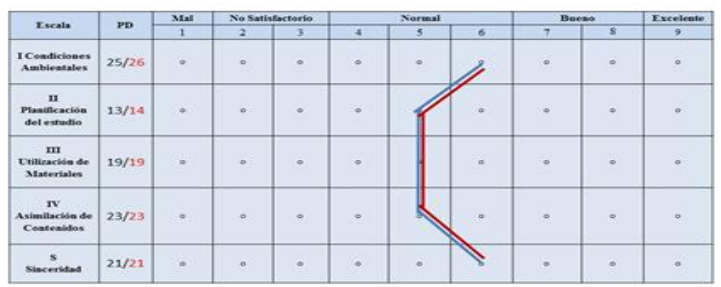

Fuente: Aplicación IHE de F. Pozar

Se aprecian los mismos hábitos de los estudiantes de la jornada nocturna tanto en la primera como en la segunda aplicación.

\subsubsection{Comparativo resultados depurados} primera y segunda aplicación jornada nocturna. El Perfil comparativo resultados depurados estudiantes jornada nocturna, primera (línea azul) y segunda (línea roja) aplicación, se aprecia en la gráfica siguiente:

Tabla 5. Perfil comparativo resultados depurados primera y segunda aplicación jornada nocturna

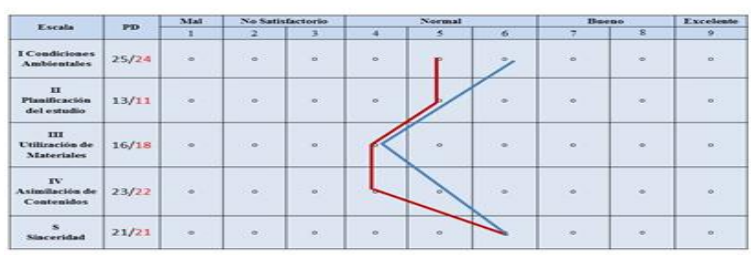

Fuente: Aplicación IHE de F. Pozar

En la segunda aplicación, los estudiantes muestran que desmejoraron sus hábitos de estudio, puesto que la curva se desplaza hacia la izquierda. Se mantiene la utilización de materiales y la sinceridad.

\subsubsection{Comparativo consolidado} resultados depurados primera y segunda aplicación jornada diurna y nocturna. Por último, se consolida toda la información, para lo cual se hace un comparativo de las dos jornadas en ambos inventarios. Los resultados son los siguientes. 
Tabla 6. Comparativo consolidado resultados depurados primera y segunda aplicación jornada diurna y nocturna

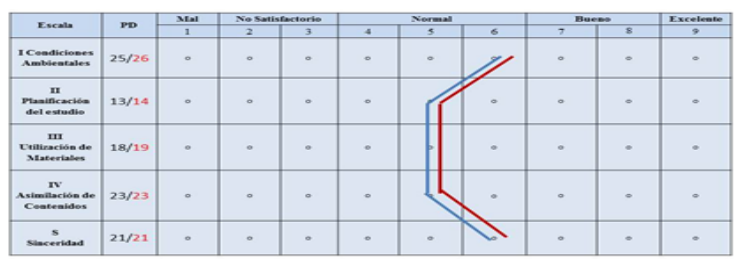

Fuente: Aplicación IHE de F. Pozar

Como se parecía, no hay variaciones significativas en los hábitos de estudio de los estudiantes de las jornadas diurna y nocturna

\section{CONCLUSIONES}

Teniendo en cuenta los objetivos del trabajo y el interés en el conocimiento de los factores que pueden influir en un mejor desempeño de los estudiantes, lo cual es tarea fundamental de la investigación en procura de evitar el fracaso escolar, la deserción y mejorar el rendimiento académico de los mismos, se concluye, de acuerdo a la aplicación del Inventario de Hábitos de estudio de Fernández Pozar en 54 estudiantes de primer semestre del programa académico de Contaduría Pública de la Universidad Francisco de Paula Santander Seccional Ocaña que sus hábitos de estudio en cuanto a condiciones ambientales en las que realizan su actividad académica, la planificación de sus estudios, la utilización de materiales y asimilación de contenidos se dan dentro del rango de la normalidad. De igual manera al aplicar una segunda medición del Inventario de Hábitos de Estudio de Fernández Pozar en este mismo grupo de estudiantes cuando cursaban su tercer semestre académico los resultados no difieren significativamente en comparación a la primera medición, sin embargo sí se presentó una deserción de 20 estudiantes correspondiente a un índice del $37 \%$ de la población inicial, lo que significa que el grupo se redujo.

Se pudo concluir que en cuanto al rendimiento académico durante las dos aplicaciones del Inventario de Hábitos de Estudio, el $74 \%$ de los estudiantes de dicha carrera, mejoraron su promedio durante el tiempo de duración del estudio, y el $18 \%$ de esta misma población, disminuyeron su promedio durante su transcurso académico. El 9\% de Ellos, mantuvieron promedio desde el inicio de su carrera hasta el segundo semestre del 2015. Lo que refleja en general un buen desempeño de dichos estudiantes en el desarrollo de sus actividades académicas.

\section{BIBLIOGRAFÍA}

Arango, M. C. (2012). Evaluación de hábitos de estudio como estrategia para la propuesta y planificación de actividades. Recuperado el 02 de marzo de 2015, de http://sedici.unlp.edu.ar/handle/10915/2 1382

Barahona, A. B. (1982). Métodos de Estudio. Bogotá: Ipler.

Barbero García, I., Holgado tello, F. P., Vila Abad, E., \& Chacón Moscoso, S. (2014). Psicothema. Recuperado el 22 de septiembre de 2015, de http://www.psicothema.com/psicothema .asp?id=3379

Barrena, b. G. (2011). Aspectos que inciden en el rendimeinto intelectual y en la eficacia en el estudio. Revista de 
trasmision del conocimiento educativo y de la salud, 223-239.

Blandon, s. P. (2011). Tecnicas de estudio utilizadas por estudiantes de noveno semestre de una facultad de enfermeria en una universidad de bogota colombia. Revista colombiana de enfermeria VOL 8, 24-34.

Cartagena Beteta, M. (2008). REICE Revista Iberoamericana sobre calidad, eficiencia y cambio en la educación. Recuperado el 12 de septiembre de 2015, de Relación entre la autoeficacia en el rendimieto escolar y los hábitos de estudio en el rendimiento académico en alumnos de secundaria : http://www.redalyc.org/pdf/551/551603 04.pdf

Colmenero Ruiz, M. J., \& Pegalajar Palomino, M. d. (s/f). Dialnet. Recuperado el 02 de marzo de 2015, de Incidencia de los hábitos de estudio en el proceso de aprendizaje de alumnos de educación primaria en la inteligencia emocional:

:///C:/Users/UFPSO/Downloads/Dialnet

IncidenciaDeLosHabitosDeEstudioEnEl ProcesoDeAprend-4644902.pdf

Contreras, K. (2008). Scielo. Recuperado el 28 de abril de 2015, de Factores Asociados al Fracaso Académico en estudiantes universitarios de Barranquilla, Colombia: http://www.scielo.org.co/pdf/psdc/n22/n 22a08.pdf

Cooper Weismann, F. J. (2011). Causan que afectan el rendimiento académico de los alumnos del primer año común de la escuela de suboficiales del ejército.
Recuperado el 12 de marzo de 2015, de http://biblioteca.universia.net/html_bura /ficha/params/title/causas-afectanrendimiento-academico-alumnos-1era\%C3\%B1o-comun-escuelasuboficiales/id/58742570.html

DeConceptos. (s/f). Concepto de Hábitos de Estudio. Recuperado el 19 de Abril de 2016 , de http://deconceptos.com/cienciassociales/habitos-de-estudio

Díaz, E. (1997). Rendimiento académico Técnicas para estudiar mejor. Bogotá: Cooperativa Editorial Magisterio.

Díaz, E. B. (1997). Rendimiento académico Técnicas para estudiar mejor. Bogotá: Cooperativa Editorial Magisterio.

Díaz, F. H. (1988). Métodos y técnicas de estudio en la Universidad. Bogotá: McGraw Hill.

Díaz, F. H. (1989). Métodos y técnicas de estudio en la universidad. Bogotá: McGraw Hill.

Díaz, F. H. (1996). Metodología del estudio, Cómo estudiar con rapidez y eficacia. Bogotá: McGraw Hill.

Enciso, V. R. (2013). Ceprosimad. Recuperado el 29 de diciembre de 2014 , de

http://ceprosimad.com/revista/habitos.p df

Erazo, O. (2 de marzo de 2012). El rendimiento académico, un fenómeno de múltiples relaciones y complejidades. Recuperado el 24 de octubre de 2016, de http://apps.umb.edu.co/revp/index.php/v anguardiapsicologica/article/view Articl $\mathrm{e} / 53$ 
Garbanzo Vargas, G. M. (2007). Factores asociados al rendimiento académico en estudiantes universitarios, una reflexión desde la calidad de la educación superior. Recuperado el 19 de Abril de 2016, de Revista Educación: http://www.redalyc.org/pdf/440/440311 03.pdf

Guerra, H., \& McCluskey, D. (1978). Cómo estudiar hoy. Madrid: Trillas S.A.

Guzmán Puentes, S. P. (2009). Repositorio Universidad Javeriana. Recuperado el 07 de enero de 2015, de http://repository.javeriana.edu.co/bitstre am/10554/425/1/edu54.pdf

H., D. F. (1988). Métodos y técnicas de estudio en la Universidad. Bogotá: McGraw Hill.

Hernández Díaz, F. (1997). Metodología de Estudio, Cómo estudiar con rapidez y eficacia. Bogotá: McGraw Hill.

Inga Arias, M., \& Inga Arias, M. (2008). Desarrollo de las Habilidades Comunicativas. San Marcos: Fondo Editorial Universidad Nacional de San Marcos Perú.

Jasso Reyes, J., Rivero Carrascal, J., Chávez Guerrero, A., Almanza Rodríguez, C. R., \& Conchas Ramírez, M. (2007). Estilos de aprendizaje y rendimiento académico en estudiantes de la Facultad de Educación Física y Ciencias del Deporte de la Universidad Autónoma de Chihuahua. Recuperado el 18 de enero de 2015, de http://afide.inder.cu/PDF/AREA\%203/F CP/FCP001.pdf

Jasso, J. R. (2007). afide. Recuperado el 18 de enero de 2015, de
http://afide.inder.cu/PDF/AREA\%203/F CP/FCP001.pdf

Jesus, J. R. (2007). afide. Recuperado el 18 de enero de 2015, de http://afide.inder.cu/PDF/AREA\%203/F CP/FCP001.pdf

López Vega, J. G. (2009). Digital library. Recuperado el 26 de Diciembre de 2015 , de file:///D:/Users/USUARIO/Desktop/Arc hivos $\% 20$ del $\% 20$ Escritorio\%202015/M aestr\%C3\%ADa\%20Consolidado/TESI S\%20DOCTORALES/habitos\%20\%20 $\% 20$ de $\% 20 \% 20$ estudio/Tesis\%20Maest ria\%20Habitos\%20de\%20Estudio $\% 20 \mathrm{~A}$ utoestima $\% 20 \mathrm{y} \% 20$ Rendimiento $\% 20 \mathrm{Ac}$ ademico\%20Jose\%20Gilberto\%20Lop

López, E. M. (1973). Cómo estudiar y cómo aprender. Buenos Aires: Kapelusz.

Martínez-Otero Pérez, V., \& Torres Barberis, L. (25 de abril de 2005). Análisis de los hábitos de estudio en una muestra de alumnos universitarios. Recuperado el 08 de diciembre de 2015, de http://www.rieoei.org/inv_edu40.htm Mira y López, E. (20 de marzo de 2013). Mediafire. Recuperado el 07 de enero de 2015, de http://www.mediafire.com/download/vc tn3cf9pynorhu/est-apr.zip

Murrieta Ortega, R., Galindo Ramírez, M. d., \& León Jiménez, J. A. (diciembre de 2014). Hábitos de Estudio en Alumnos Normalistas y su Incidencia en el Rendimiento Académico. Recuperado el 31 de mayo de 2015, de https://scholar.google.com.co/scholar?q $=\mathrm{H} \% \mathrm{C} 3 \%$ Albitos+de+estudio+en+alu mnos+normalistas $+\mathrm{y}+\mathrm{su}+\mathrm{incidencia}+\mathrm{en}$ +el+rendimiento+acad\%C3\%A9mico\& 
btnG $=\&$ hl=es\&as_sdt=0\%2C5\&as_ylo $=2011 \&$ as_vis $=1$

Murrieta, R. (julio de 2014). Revista Iberoamericana de Producción Académica y Gestión Educativa. Hábitos de Estudio en alumnos normalistas y su incidencia en el rendimiento académico. Recuperado el 31 de mayo de 2015, de https://scholar.google.com.co/scholar?q $=\mathrm{H} \% \mathrm{C} 3 \%$ Albitos+de+estudio+en+alu mnos+norma

Oré ortega, R. Z. (2012). Digital Library. Recuperado el 27 de Diciembre de 2015 , de http://cybertesis.unmsm.edu.pe/bitstrea m/cybertesis/2738/1/Or\%C3\%A9_or.pd $\mathrm{f}$

Ortega mollo, B. V. (2012). repositorio usil. Recuperado el 27 de Diciembre de 2015 , de

http://repositorio.usil.edu.pe/wpcontent/uploads/2014/07/2012_Ortega_ $\mathrm{H} \% \mathrm{C} 3 \%$ A1bitos-de-estudio-yrendimiento-acad\%C3\%A9mico-enestudiantes-de-segundo-de-secundariade-una-instituci\%C3\%B3n-educativadel-Callao.pdf

Posada Jeancjaquez, J. Á. (13 de Julio de 2012). Tecnicrim. Recuperado el 18 de Abril de 2016, de http://www.tecnicrim.co.cu/wpentrada.a spx?6,29,

Pozar, F. F. (1987). Tea ediciones. Recuperado el 14 de 10 de 2014, de http://www.web.teaediciones.com/Ejem plos/IHE_MANUAL_2014_extracto.pd $\mathrm{f}$

Pozar, F. F. (s/f). teaediciones. Recuperado el 14 de 10 de 2014, de
http://www.web.teaediciones.com/Ejem plos/IHE_MANUAL_2014_extracto.pd $\mathrm{f}$

Puentes, S. P. (noviembre de 2009). Universidad Javeriana. Recuperado el 18 de enero de 2015, de http://www.javeriana.edu.co/biblos/tesis /educacion/tesis81.pdf

Rampirez, A. V. (octubre de 2009). Elibro. Recuperado el 18 de diciembre de 2015, de

ProQuuest: http://site.ebrary.com/lib/ufpsosp/reader .action?docID $=10345271 \& \mathrm{p} 00=\mathrm{h} \% \mathrm{C} 3$ $\%$ A1bitos+de+estudio\&ppg $=9$

Real Academia Española. (2010). Recuperado el 17 de noviembre de 2015, de http://www.rae.es/

Reyes, J. J. (s.f.). afide. Recuperado el 18 de enero de 2015, de http://afide.inder.cu/PDF/AREA\%203/F CP/FCP001.pdf

Ríos, V., \& Ramos Enciso, D. (2013). Hábitos de Estudio y Rendimiento Académico en los estudiantes de carreras profesionales de ingeniería, ecoturismo y educación de la universidad Nacional Amazónica de Madre de Dios, ciudad de Puerto Maldonado, Perú. Recuperado el 7 de Noviembre de 2014, de http://ceprosimad.com/revista/hábitos.p df

Sarabia Granda, E. A. (2012). www.dspace.uce.edu.ec. Recuperado el 27 de Diciembre de 2015, de http://www.dspace.uce.edu.ec/bitstream /25000/1900/1/T-UCE-0010-258.pdf

Scielo. (s.f.). Obtenido de Factores Asociados al Fracaso A: 
http://www.scielo.org.co/pdf/psdc/n22/n 22a08.pdf

Staton, T. F. (1979). Cómo Estudiar. México: Trillas.

Toro, E. (abril de 2007). Relación de los Hábitos de Estudio y el rendimiento académico. Recuperado el 28 de diciembre de 2014, de http://site.ebrary.com/lib/ufpsosp/reader .action?docID $=10345271 \& \mathrm{p} 00=\mathrm{h} \% \mathrm{C} 3$

$\%$ Albitos+de+estudio\&ppg $=9$

Torres Narváez, M., Tolosa Guzmán, I., Urrea González, C., \& Monsalve Robayo, A. (diciembre de 2009). Inventario de hábitos de estudio en una clase para toma de decisiones deestudiantes de fisioterapia. Recuperado el 8 de diciembre de 2015, de

http://www.scielo.org.co/scielo.php?scri $\mathrm{pt}=\mathrm{sci} \_$arttext\&pid=S169272732009000300006

Triviños, M. (2011). nfoques de aprendizaje y hábitos deestudio en estudiantes universitarios de primer años de tres carreras de la universidad Mayor Temuco,. Revista Educativa Hekademos, 37-46.

Valle, A., Rodríguez, S., Cabanach, R. G., Núñez, J. C., González Pinedo, J. A., \& Rosario, P. (septiembre de 2009). Diferencias en rendimiento académico según los niveles de las estrategias cognitivas y de las estrategias de autorregulación. Recuperado el 31 de mayo de 2015, de http://www.summapsicologica.cl/index. $\mathrm{php} / \mathrm{summa} /$ article/view/60/59

Vásquez Vásquez, Á. W., \& Vásquez Vasquez, S. L. (2012). Revista
Científica Universidad Señor de Sipán. Recuperado el 26 de Diciembre de 2015, de http://servicios.uss.edu.pe/ojs/index.php /tzh/article/view/57

Vélez Ramírez, A. (octubre de 2009). ProQuest Ebrary- La Adquisición de hábitos como finalidad de la educación superior. Recuperado el 14 de septiembre de 2015, de http://site.ebrary.com/lib/ufpsosp/reader .action?docID=10345271\&p00=h\%C3 $\%$ Albitos+de+estudio\&ppg $=9$ 